\title{
Serum biomarker CA $15-3$ as predictor of response to antifibrotic treatment and survival in idiopathic pulmonary fibrosis
}

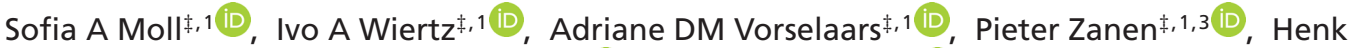 \\ JT Ruven ${ }^{\ddagger}, 2$, Coline HM van Moorsel ${ }^{*,+, 1}$ (iD) \& Jan C Grutters ${ }^{\ddagger}, 1,3$ (iD) \\ ${ }^{1}$ Department of Pulmonology, Centre for Interstitial Lung Diseases, St. Antonius Hospital, Koekoekslaan 13435 CW, Nieuwegein, \\ The Netherlands \\ ${ }^{2}$ Department of Clinical Chemistry, St. Antonius Hospital, Koekoekslaan 13435 CW, Nieuwegein, The Netherlands \\ ${ }^{3}$ Division Heart \& Lungs, University Medical Center Utrecht, Heidelberglaan 1003584 CX, Utrecht, The Netherlands \\ *Author for correspondence: s.moll@antoniusziekenhuis.nl \\ $\ddagger$ Authors contributed equally
}

\begin{abstract}
Aim: Cancer antigen 15-3 (CA 15-3) is a baseline biomarker in idiopathic pulmonary fibrosis (IPF), but its value during follow-up is unknown. Materials and methods: Associations between serum CA 15-3 and pulmonary function tests during 1-year follow-up were evaluated by a mixed model in 132 IPF treated with pirfenidone or nintedanib. Results: Increased baseline (median: $56 \mathrm{kU} / \mathrm{l}$ ) and follow-up CA 15-3 levels were inversely associated with forced vital capacity and diffusing capacity of the lung for carbon monoxide (estimates respectively: -5.21 and $-4.69 ; \mathrm{p}<0.001$ ). Baseline and 6-month CA 15-3 above 58.5 (hazard ratio: $1.67 ; p=0.031$ ) and $50.5 \mathrm{kU} / \mathrm{l}$ (hazard ratio: $2.99 ; \mathrm{p}<0.001$ ), respectively, showed impaired survival compared with lower levels. Conclusion: CA $15-3$ is associated with pulmonary function test during followup in IPF on antifibrotic treatment. Higher (follow-up) values are related with poor survival. Therefore, CA 15-3 is a promising follow-up biomarker in IPF.
\end{abstract}

First draft submitted: 25 March 2020; Accepted for publication: 29 May 2020; Published online: 17 September 2020

Keywords: anti-fibrotic therapy $\bullet$ cancer antigen 15-3 • idiopathic pulmonary fibrosis $\bullet$ interstitial lung disease $\bullet$ prognostic biomarker $\bullet$ survival

Idiopathic pulmonary fibrosis (IPF) is a chronic progressive, fibrosing interstitial lung disease (ILD) of unknown cause [1]. IPF patients are predominately male and the disease is characterized by progressive dyspnea [2-4]. IPF is the result of activated fibroblast-myofibroblasts after repetitive damage, which play an important role in tissue remodeling and injury of alveolar type II pneumocytes. As a result, pneumoproteins are secreted by type II pneumocytes [5,6].

The prognosis is extremely poor, with a median survival of $2-3$ years after diagnosis, although this is very heterogeneous [7]. Therefore, it is difficult to predict the exact prognosis [8]. Currently, the 6-minute walking test is used to measure exercise tolerance as reflection of disease status in IPF [9]. Furthermore, the GAP index that is based on gender, age and pulmonary function tests (PFTs) is used for predictive purposes [10]. More than $5 \%$ decline in forced vital capacity (FVC) and 15\% decline in diffusing capacity of the lung for carbon monoxide (DLco) per year correlate with increased mortality in IPF [10-12]. However, PFT declines are not specific enough to predict individual prognosis and PFTs may be invasive in patients with a severe condition $[9,10,12,13]$.

Possibly, blood biomarkers such as serum pneumoproteins have a more accurately predictive value in IPF, as they reflect the process of remodeling and injury of the lungs [14]. A well-studied serum biomarker is CC-chemokine ligand 18 (CCL18). It was demonstrated that increased baseline levels are associated with high mortality in IPF [15]. Change in markers of the extracellular matrix was associated with disease progression and mortality in IPF as well $[4,16]$. A suggested pneumoprotein is Krebs von den Lungen antigen (KL-6), an epitope of the mucin 1 protein that is expressed at alveolar type II pneumocytes [17]. Mucins are recognized as biomarkers, as they play a role in cell growth and tissue remodeling, compatible with the processes as seen in IPF [18]. Increased baseline KL-6 predicted 
a higher risk on acute exacerbations and increased mortality in IPF at baseline and during follow-up [17,19,20]. KL-6 change was found to be an indicative of nintedanib response in IPF patients, as KL-6 levels declined during treatment and were associated with PFT [21]. However, KL-6 analysis is expensive and not available on a large scale. The epitope cancer antigen 15-3 (CA 15-3), also located at mucin 1, is considered to be an alternative biomarker for KL-6 [14,22,23]. It is a minimal invasive measurement available on a large scale and less expensive than the KL-6 ELISA kit [14]. CA 15-3 was originally used as a tumor-associated biomarker in neoplasms including lung carcinoma and breast cancer [24,25], but is also associated with fibroblast activity, progression of pulmonary fibrosis and fibrotic characteristics on high-resolution computed tomography (HRCT) scans [26]. The role of CA 15-3 as a prognostic biomarker has been confirmed in fibrotic ILDs including hypersensitivity pneumonitis (HP), systemic sclerosis (Ssc-ILD) and rheumatoid arthritis (RA-ILD) [22,26-35]. Elevated baseline CA 15-3 levels were found in IPF and were higher compared with sarcoidosis, Ssc-ILD and healthy subjects [14,26]. Furthermore, a negative correlation between serum CA 15-3 and FVC was found in progressive IPF patients referred for lung transplantation, whereas CA 15-3 was negatively associated with DLco in Ssc-ILD [30,36].

We previously demonstrated that (early) CA 15-3 change correlated inversely with (future) PFT change in HP [33]. However, CA 15-3 change over time has not been described in IPF. Furthermore, it is unknown whether CA 15-3 levels reflect PFT or treatment response of antifibrotic drugs, such as nintedanib and pirfenidone. These pharmaceuticals lead to a reduction of FVC decline in the majority of IPF patients [12,37]. As fibrotic activity is reflected by CA 15-3 secretion by type II alveolar pneumocytes, it is possible that CA 15-3 reflects drug effectiveness as well.

This retrospective study investigated the prognostic value of serum CA 15-3 in IPF. We hypothesized that CA 15-3 during follow-up was inversely associated with PFT in IPF patients using antifibrotic therapy. Furthermore, we evaluated if CA 15-3 predicted PFT outcome and survival.

\section{Methods}

A retrospective cohort study was performed at the St. Antonius ILD Centre of Excellence Nieuwegein, a tertiary referral centre for ILD in The Netherlands. Medical archives of patients diagnosed with IPF between February 2012 and February 2019 were examined. Patients were included if therapy with anti-fibrotic drugs, in other words, nintedanib or pirfenidone, was initiated within 6 months from date of diagnosis. Patients without antifibrotic treatment or initiation of therapy after 6 months were excluded. All patients gave written informed consent for retrospective study purposes. The study was approved by the St Antonius institutional review board (registration number R05-08A and W14.056).

Patients were diagnosed in a multidisciplinary discussion between a radiologist, ILD pulmonologist and if needed a pathologist, according to ATS/ERS/JRS/ALAT criteria for IPF: a pattern of usual interstitial pneumonia (UIP) found on HRCT and if available, in histological lung biopsy. Patients with a UIP secondary to known causes, including environmental exposures, collagen disease and drug-induced lung fibrosis, were excluded.

IPF patients on antifibrotic treatment visited the outpatient clinic every 3 months according to a standard protocol. Serum CA 15-3 was routinely measured on an immunochemistry analyzer (Cobas e601, Roche Diagnostics Ltd., Rotkreuz, Switzerland) according to the manufacturer's instructions. Samples were centrifuged and analyzed within $3 \mathrm{~h}$ after serum sampling. Values above $30 \mathrm{kU} / \mathrm{l}$ were considered as elevated. CA 15-3 levels measured at date of diagnosis (baseline) and during follow-up at 3, 6 and 12 months were included for analysis.

PFTs were routinely performed at diagnosis and during therapy. Spirometry and diffusion capacity for carbon monoxide were executed at the St Antonius Hospital by a Jaeger System (provided by CareFusion Ltd., Houten, The Netherlands) and carried out according to ERS recommendations [11]. FVC percent predicted and DLco percent predicted measured at baseline and at 3,6 and 12 months of follow-up were included for analysis.

Patients with available biomarker and PFT measured at date of diagnosis and with at least one follow-up measurement were suitable for the study. Follow-up was censored after 72 months and ended if patients were referred to their own physician, if patients underwent a lung transplantation and in case of death. Patients were excluded from analysis if solely baseline measurements were available.

Other baseline variables evaluated in the study were age, sex, smoking status and GAP index. In addition, characteristics on HRCT and in histopathological lung biopsies were described. Characteristics were classified according to the most recent American Thoracic Society/European Respiratory Society (ATS/ERS) recommendations as a pattern of UIP, probable UIP, indeterminate UIP or alternative diagnosis [38]. 


\section{Statistical analysis}

Baseline characteristics were expressed as mean and standard deviation (SD) for normally distributed data or as median and interquartile range (IQR) for non-normally distributed data or as numbers and percentages. Independent sample $t$-test was executed to evaluate continuous data between the nintedanib and pirfenidone group, whereas Chi-square test was used to assess dichotomous data. A repeated measures linear mixed model was used to analyze the pulmonary function over time, as function of the observed CA 15-3 outcomes. In such an analysis for each patient, a regression equation is obtained depicting an intercept and the changes in PFT outcomes between the fixed observation points. In this analysis, the last observation serves as reference, while the intercept is the (PFT-) value at the start of the observation period $(\mathrm{T}=0)$. The correlation between successive observations needs to be taken into account: we used as starting point an autoregressive correlation structure and refined the analyses based on -2LL comparisons. The individual outcomes are summarized into cohort describing regression equations:

- $\operatorname{FVC}(\%$ of pred $)=\alpha+\beta_{1}{ }^{*}$ CA 15-3 $(\ln )+\beta_{2}{ }^{*}$ time $+\beta_{3}{ }^{*}$ treatment $+\beta_{4}{ }^{*}$ time ${ }^{*}$ treatment;

- DLco $(\%$ of pred $)=\alpha+\beta_{1}{ }^{*}$ CA $15-3(\ln )+\beta_{2}{ }^{*}$ time $+\beta_{3}{ }^{*}$ treatment $+\beta_{4}{ }^{*}$ time ${ }^{*}$ treatment, where 'time' reflects the observation points.

The last term in these equations is a time*treatment interaction, which tries to find a difference in time trends between the two treatment groups. We in-transformed CA-15-3 data to obtain a more suitable distribution.

Survival time was expressed in months from date of diagnosis. The optimal cut-off values of baseline and follow-up CA 15-3 levels were determined using receiver operating characteristic (ROC) curves on progression of pulmonary function. FVC progression was defined as an absolute FVC decline of at least $5 \%$ after 1 year, whereas DLco progression was defined as an absolute DLco decline of 15\%. Survival plots were created using Kaplan-Meier curves. A p-value less than 0.05 was considered as statistically significant. The statistical analysis was performed by software IBM SPSS 24.0. Graphs were drafted in GraphPad Prism 8.3.

\section{Results}

\section{Baseline characteristics}

A total of 132 patients diagnosed with IPF between 2012 and 2019, treated with nintedanib or pirfenidone within 6 months after diagnosis were identified. All patients had biomarker and PFT data available at diagnosis and at least on one follow-up time point. Table 1 summarizes the demographics of these patients at date of diagnosis. Subjects were predominately male (75.8\%) with mean age 68.6 years (SD: 7.8) and mean GAP index of 3.95 (SD: 1.3). Overall, baseline serum CA 15-3 was increased (median: $56.0 \mathrm{kU} / \mathrm{l}$; IQR: 34.0-89.8). FVC (\% pred) was considerably high (79.5; SD: 18.7), whereas low DLco (\% pred) values were observed (40.9; SD: 12.3). A radiological UIP pattern was predominately seen $(63.6 \%)$, followed by probable UIP $(31.8 \%)$ and indeterminate UIP (4.5\%; Table 1). Available lung biopsies of patients with a radiological probable UIP $(\mathrm{n}=13)$ and indeterminate UIP ( $n=6)$ all demonstrated a histological UIP pattern. No baseline differences were observed between pirfenidoneand nintedanib-treated patients (Table 1). No statistical differences were found in median baseline CA 15-3 levels between patients with a radiological UIP, probable UIP and indeterminate UIP pattern ( $\mathrm{p}=0.508$, data not shown), nor between patients with a histological UIP and probable UIP pattern ( $\mathrm{p}=0.901$, data not shown).

\section{CA $15-3$ as marker for PFT}

Mean follow-up duration was 47.0 months (SD: 14.9). The results of the linear mixed model analysis to evaluate the effect of CA 15-3 on PFT outcome over time are listed in Table 2. No significant differences in time trends were found between the pirfenidone and nintedanib groups for FVC $(p=0.239)$ and DLco $(p=0.820)$ outcomes and we removed the time*treatment interaction from the analysis.

In the linear mixed model, the 12-month measurement of PFT was set as reference point (0) of the effect of time on previous PFT outcomes. The estimates at baseline, 3 and 6 months were determined in comparison to the reference point. Overall, a significant effect of time on FVC $(p=0.030)$ and DLco $(p<0.001)$ values is evident, indicating that FVC and DLco values declined over time.

An overview of the regression coefficients is listed in Table 2. CA 15-3 (ln) measurements were inversely associated with the FVC (an estimated drop of 5.21\% predicted per unit increase in In-CA 15-3; p < 0.001) and DLco (an estimated drop of $4.69 \%$ predicted per unit increase in ln-CA 15-3; $\mathrm{p}<0.001$ ). For illustration, the course 
Table 1. Characteristics of patients with idiopathic pulmonary fibrosis at time of start of therapy.

\begin{tabular}{|c|c|c|c|c|}
\hline \multirow[t]{2}{*}{ Parameters } & \multirow{2}{*}{$\begin{array}{l}\text { Subjects } \\
\text { All }\end{array}$} & \multicolumn{3}{|c|}{ Treatment } \\
\hline & & Pirfenidone & Nintedanib & p-value \\
\hline n (\%) & 132 & 70 & 62 & \\
\hline Age (years) & $68.6(7.8)$ & $68.7(8.0)$ & $68.5(7.8)$ & 0.928 \\
\hline Sex (M), n (\%) & $100(75.8)$ & $52(74.3)$ & $48(77.4)$ & 0.675 \\
\hline Current/exsmoker, no (\%) & $102(75.8)$ & $57(81.4)$ & $48(77.4)$ & 0.226 \\
\hline GAP index, mean (SD) & $3.95(1.3)$ & $3.96(1.2)$ & $3.95(1.4)$ & 0.394 \\
\hline \multicolumn{5}{|l|}{ Lung biomarker } \\
\hline $\begin{array}{l}\text { - CA 15-3 (kU/I), median } \\
\text { (IQR) }\end{array}$ & $56.0(34.0-89.8)$ & $55.5(33.0-92.3)$ & $58.0(34.8-88.3)$ & 0.659 \\
\hline \multicolumn{5}{|l|}{ Pulmonary function } \\
\hline - FVC (\%, pred), mean (SD) & $79.5(18.7)$ & $78.0(18.7)$ & $81.3(18.8)$ & 0.304 \\
\hline - DLco (\%, pred), mean (SD) & $40.9(12.3)$ & $40.0(11.4)$ & $41.8(13.3)$ & 0.462 \\
\hline \multicolumn{5}{|l|}{ HRCT scan, n (\%) } \\
\hline- UIP & $84(63.6)$ & $47(67.1)$ & $37(59.7)$ & 0.374 \\
\hline - Probable UIP & $42(31.8)$ & $21(30.0)$ & $21(33.9)$ & 0.634 \\
\hline - Indeterminate UIP & $6(4.5)$ & $2(2.9)$ & $4(6.5)$ & 0.415 \\
\hline - Alternative diagnosis & - & - & - & - \\
\hline \multicolumn{5}{|l|}{ Histopathology, n (\%) ${ }^{\dagger}$} \\
\hline- UIP & $27(96.4)$ & $12(92.3)$ & $15(100)$ & 0.464 \\
\hline - Probable UIP & $1(3.6)$ & $1(7.7)$ & - & 0.464 \\
\hline - Indeterminate UIP & - & - & - & - \\
\hline - Alternative diagnosis & - & - & - & - \\
\hline \multicolumn{5}{|c|}{$\begin{array}{l}\text { Characteristics on HRCT and in histopathological lung biopsies were classified according to ATS/ERS } 2018 \text { recommendations. } \\
\operatorname{t}_{n}=28 \text {. } \\
\mathrm{p}<0.05 \text {; difference in variables between pirfenidone and nintedanib groups. } \\
\text { ATS/ERS: American Thoracic Society/European Respiratory Society; CA 15-3: Cancer antigen 15-3; DLco: Diffusing capacity of the lung for carbon monoxide, in percentage of predicted; } \\
\text { FVC: Forced vital capacity, in percentage of predicted; GAP index: IPF severity index based on gender, age and pulmonary function test; HRCT: High-resolution computed tomography; } \\
\text { IQR: Interquartile range; SD: Standard deviation; UIP: Usual interstitial pneumonia. }\end{array}$} \\
\hline
\end{tabular}

of pulmonary function and CA 15-3 measurements over time as calculated by the equation are shown for a pirfenidone- and a nintedanib-treated patient (Figure 1).

CA $15-3$ as predictor of survival

Within the study period, 74 out of 132 patients died (56.1\%). Overall, median survival was 33 months (IQR: 21.339.0). ROC curves were used to define cut-off CA 15-3 values to predict FVC progression and DLco progression. ROC curves of CA 15-3 levels and CA 15-3 change demonstrated no significant areas under the curve (AUCs) for FVC progression. Contrary, the AUC was significant for DLco progression $(0.650 ; \mathrm{p}=0.031)$, with baseline CA $15-3$ level of $58.5 \mathrm{kU} / 1$ identified as the value with the highest sum of sensitivity and specificity (1.323). Patients were categorized as having low-baseline CA 15-3 (CA 15-3 <58.5) or high-baseline CA 15-3 (CA $15.3>58.5$ ) levels. Survival analysis demonstrated a significantly impaired survival of patients with high-baseline CA 15-3 (median: 29 months) compared with lower levels (median: 50 months; hazard ratio: 1.67; 95\% CI: 1.05-2.65; $\mathrm{p}=0.031$, Figure 2A). No significant differences were found in radiological and histological characteristics between patients with baseline CA $15-3$ levels above $58.5 \mathrm{kU} / \mathrm{l}$ and those with lower levels (data not shown).

The ROC curve of the 6-month CA 15.3 levels demonstrated an AUC of 0.694 ( $\mathrm{p}=0.006)$ with a value of $50.5 \mathrm{kU} / 1$ determined as cut-off value for predicting DLco progression. Survival was significantly deteriorated in patients with 6-month CA 15-3 levels above $50.5 \mathrm{kU} / 1$ (median: 28.0 months) compared with those with lower levels (median: 65 months; hazard ratio: 2.99; 95\% CI: 1.74-5.13; p < 0.001, Figure 2B).

Survival rates between pirfenidone and nintedanib-treated IPF patients showed no significant differences (data not shown). 


\begin{tabular}{|c|c|c|c|c|}
\hline Parameters & Estimate & SE & $p$-value & $95 \% \mathrm{Cl}$ \\
\hline Intercept & 100.69 & 6.00 & $<0.001$ & $88.89-112.48$ \\
\hline \multicolumn{2}{|l|}{ Time (months) } & & \multicolumn{2}{|l|}{0.030} \\
\hline - Baseline & 2.22 & 0.85 & 0.011 & $0.51-3.88$ \\
\hline-6 & 1.34 & 0.74 & 0.074 & -0.13 to -2.81 \\
\hline$-12^{\dagger}$ & 0 & - & - & - \\
\hline \multicolumn{5}{|l|}{ Treatment } \\
\hline - Pirfenidone & -3.99 & 3.38 & 0.239 & $-10.67-2.68$ \\
\hline - Nintedanib $\ddagger$ & 0 & - & - & - \\
\hline - Baseline & 5.21 & 0.73 & $<0.001$ & $3.78-6.64$ \\
\hline-3 & 2.72 & 0.68 & $<0.001$ & $1.38-4.06$ \\
\hline-6 & 2.24 & 0.57 & $<0.001$ & $1.11-3.37$ \\
\hline$-12^{\dagger}$ & 0 & - & - & - \\
\hline \multicolumn{5}{|l|}{ Treatment } \\
\hline - Pirfenidone & 0.48 & 2.07 & 0.820 & $-3.62-4.57$ \\
\hline - Nintedanib & 0 & - & - & - \\
\hline CA 15-3 (In) & -4.69 & 1.05 & $<0.001$ & -6.75 to -2.63 \\
\hline \multicolumn{5}{|c|}{ 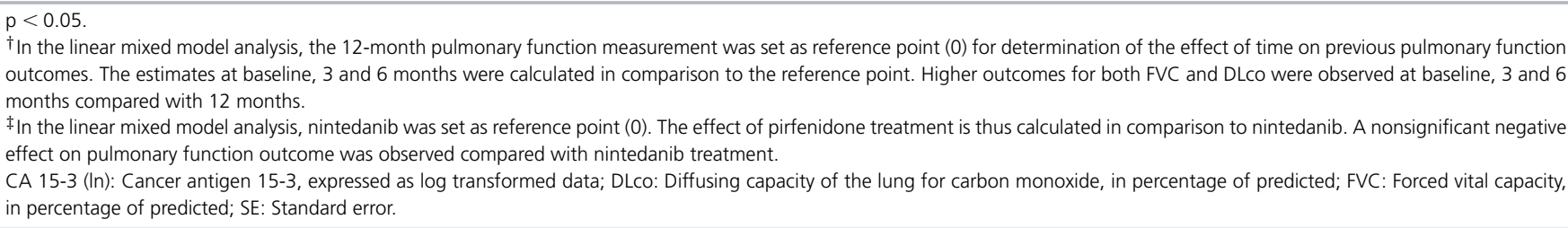 } \\
\hline
\end{tabular}

\section{Discussion}

In this study, the predictive value of serum CA 15-3 in IPF patients was evaluated. CA 15-3 was inversely associated with FVC (\% pred) and DLco (\% pred) during 1-year follow-up in pirfenidone- and nintedanib-treated patients. Furthermore, increased baseline and 6-month CA 15-3 levels predicted a significantly worse survival compared with lower levels. Therefore, serum CA 15-3 could be implemented as a prognostic biomarker in IPF.

Increased baseline CA 15-3 levels were found in IPF patients compared with healthy controls in previous research [26]. In our study, CA 15-3 was strongly associated with pulmonary function during follow-up, in particular with DLco. Increased CA 15-3 levels and associations with severe lung involvement have been demonstrated in fibrotic ILDs including sarcoidosis, HP, Ssc-ILD and RA-ILD [22,26,27,30-35]. Negative associations between baseline DLco and CA 15-3 were found in patients with sarcoidosis and Ssc-ILD [30,39]. In contrast to our results, a weak positive association of CA 15-3 and FVC was found in IPF patients referred for lung transplantation, although selection bias of more progressive patients might have occurred in this study [36]. It was recently demonstrated that CA 15-3 is inversely associated with PFT in HP [33]. Our results add to these findings that CA 15-3 appears to be a predictive biomarker on baseline and during follow-up for PFT outcome in IPF as well.

Increased CA 15-3 levels above 50.8 and $50.5 \mathrm{kU} / \mathrm{l}$ at baseline and 6 months, respectively, were associated with poor survival compared with lower levels in our present study. Several IPF studies demonstrated decreased survival rates in patients with high-baseline KL-6 compared with lower levels as well [19,40,41]. Moreover, IPF patients with positive KL- 6 change had a worsened survival and were associated with greater FVC declines compared with unchanged or negative KL-6 changes [40]. Our study adds to previous findings that in addition to KL-6, increased CA 15-3 levels associate with decreased PFT outcome and survival during follow-up in IPF. 

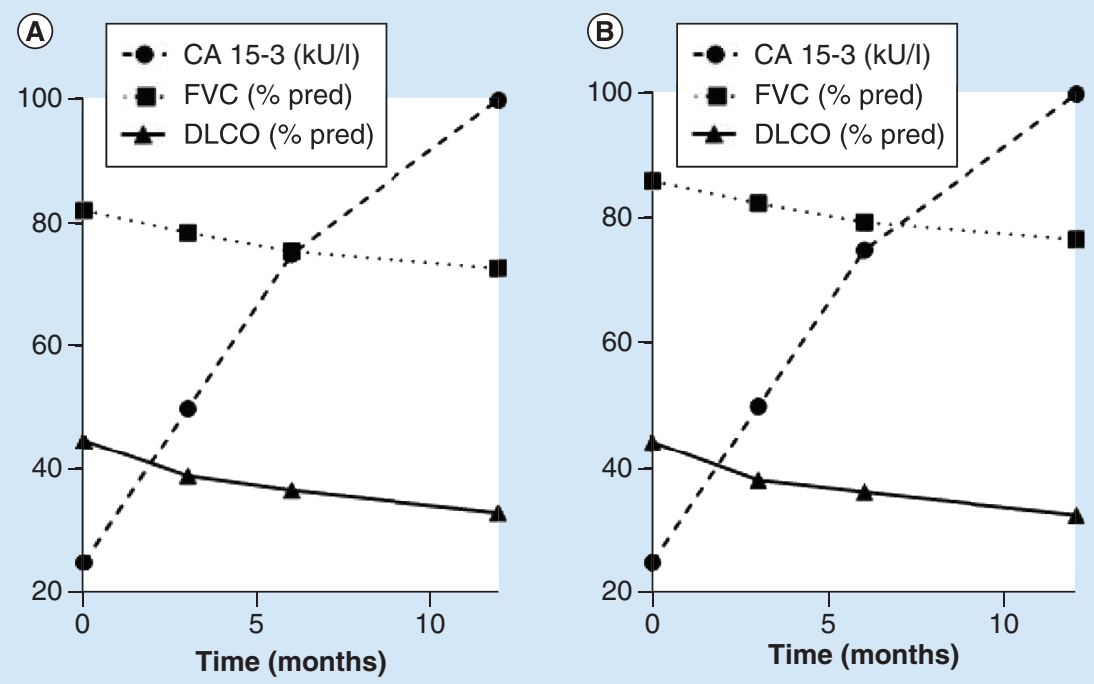

Figure 1. The course of CA 15-3 (kU/I), FVC (\% pred) and DLco (\% pred) over time. (A) The course of CA 15-3 (kU/I), FVC (\% pred) and DLco (\% pred) as illustrated by the linear mixed model analyses for a pirfenidone-treated patient. (B) The course of CA 15-3 (kU/l), FVC (\% pred) and DLco (\% pred) as illustrated by the linear mixed model analyses for a nintedanib-treated patient.
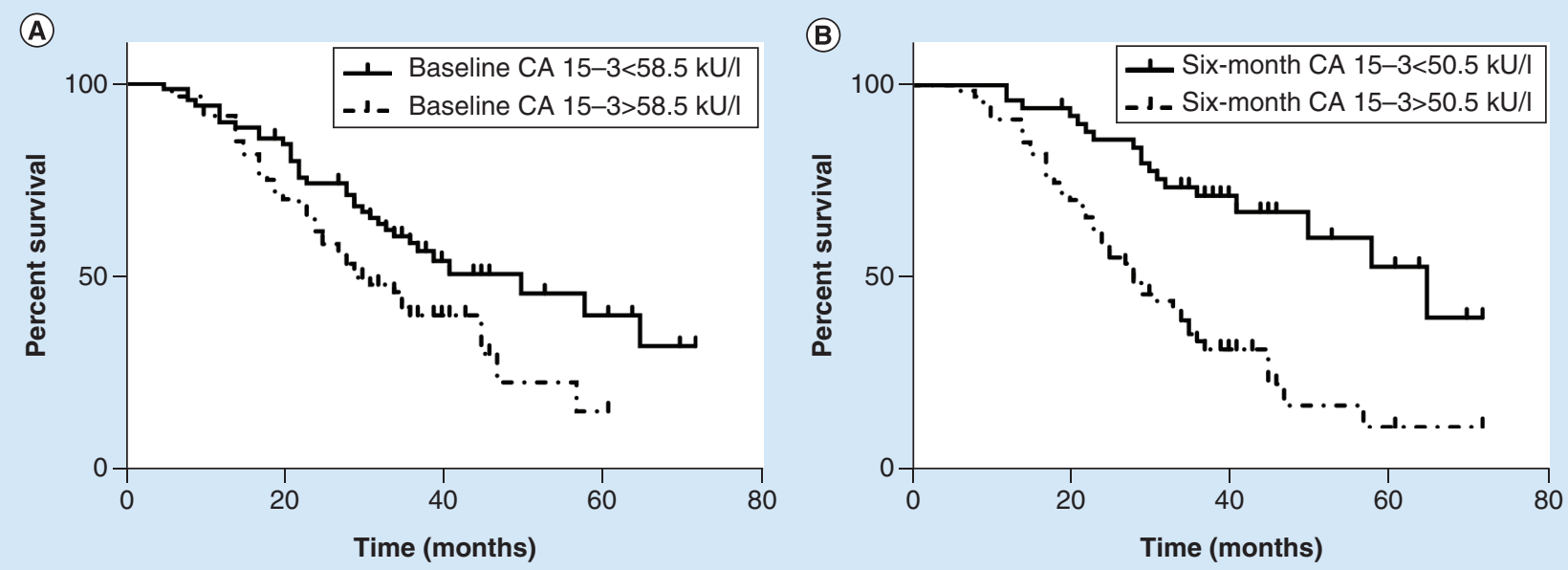

Figure 2. Survival curves of IPF patients based on CA 15-3 outcome. (A) Kaplan-Meier curve showing significantly impaired survival in patients with baseline CA 15-3 levels above $58.5 \mathrm{kU} / \mathrm{I}$ (median: 29 months) compared with baseline CA 15-3 levels below 58.5 kU/I (median: 50 months; hazard ratio: 1.67; 95\% Cl: 1.05-2.65; $\mathrm{p}=0.031$ ). (B) Kaplan-Meier curve showing significantly impaired survival in patients with 6-month CA 15-3 levels above $50.5 \mathrm{kU} / \mathrm{I}$ (median: 28.0 months) compared with 6-month CA 15-3 levels below $50.5 \mathrm{kU} / \mathrm{I}$ (median: 65 months; hazard ratio: $2.99 ; 95 \% \mathrm{Cl}: 1.74-5.13 ; \mathrm{p}<0.001$ ).

CA 15-3 has been associated with progression of pulmonary fibrosis and fibrotic characteristics on HRCT in IPF [26]. In our study, we did not find differences in baseline CA 15-3 levels between IPF patients with a UIP, probable UIP or indeterminate UIP pattern on HRCT and/or in lung biopsies. Possibly, CA 15-3 levels do not specifically differentiate between the various interstitial findings on baseline, but could have a prognostic value for future radiological and/or histological progression. A similar phenomenon has been described in Ssc-ILD, in which patients with worsening of the interstitial HRCT score during follow-up had significant higher baseline CA 15-3 levels compared with patients with a stable interstitial HRCT score [30,31]. 
This retrospective cohort study has some limitations by selecting IPF patients in a tertiary ILD center. Solely patients treated with antifibrotic therapy and with available follow-up CA 15-3 and PFT measurements were selected for analysis. Outcomes on PFT and survival might be impaired due to selection of more severe patients. Diagnosis and follow-up of patients was conducted by experienced ILD pulmonologists and according to a standardized protocol. As a result, a large sample size was obtained with a considerable follow-up period and limited missing data.

According to ATS/ERS recommendations, the change in FVC is currently used as most important predictor in IPF [11]. We are the first to demonstrate the predictive value of serial CA 15-3 levels on FVC, DLco and survival in a large cohort of IPF treated with antifibrotic therapy. We identified cut-off CA 15-3 values based on DLco progression by AUCs. The nonsignificant AUC for FVC progression in our study most probably originated in a lower percentual decline of the FVC (2.22\%) compared with DLco (5.31\%). In a follow-up study of nintedanibtreated IPF patients, KL- 6 was confirmed to predict DLco progression and indicative of therapy response [21]. As associations between CA 15-3 and DLco were seen both in nintedanib and pirfenidone patients in our study, it could be hypothesized that CA 15-3 might reflect therapy response of antifibrotic treatment in IPF as well.

It is thought that DLco reflects the permeability of the alveolar surface [42]. KL-6 was demonstrated to be a representative marker for increased alveolar-capillary permeability [43]. Possibly, CA 15-3 is also more a reflection of alveolar-capillary permeability, and thus reflects DLco, due to lung injury than a reflection of fibroblast activity. As the condition in IPF patients declines in progressive disease, PFT may be invasive and even unreliable if maximal performance cannot be reached. Blood sampling is less invasive and more objective than PFT. Measurement of CA 15-3 could be used as an indicator of pulmonary function and treatment response of antifibrotic therapy in IPF.

For future research, it would be interesting to investigate if CA 15-3 has prognostic value for worsening of interstitial HRCT findings during follow-up, as seen in Ssc-ILD [30,31]. Furthermore, evaluation of associations between CA 15-3 and dyspnea severity is an interesting research topic, as KL-6 was found to be associated with dyspnea severity, decreased physical activity and decreased walking distance in IPF [44,45]. Moreover, it would be interesting to evaluate in future research whether CA 15-3 is associated with other biomarkers such as CCL18 and lactate dehydrogenase (LDH) or tumor-associated biomarkers. Strong associations between CA 15-3 and tumorassociated biomarkers CEA, CA 125 and CA 19-9 were observed in RA-ILD and idiopathic interstitial pneumonia (IP) without underlying carcinoma [26,35,46-48]. As mucin 1 upregulation is associated with smoking [49], it would be relevant for clinical interpretation of CA 15-3 to compare levels between smoking and nonsmoking ILD patients. For clinical practice, it would be valuable to assess whether CA 15-3 levels predict IPF exacerbations, as elevated KL-6 levels were associated with a short follow-up period before the onset of an acute exacerbation of IPF as well [20]. In practice, preventive preparations for an exacerbation of IPF could then be taken into account when CA 15-3 is increasing [50]. Several trials have recently been published on nintedanib as treatment in Ssc-related ILD and progressive ILDs [51,52]. It would be interesting to investigate if CA 15-3 is indicative for nintedanib response in other ILD-related disease as well. In addition, it would be valuable to further assess CA 15-3 as biomarker for treatment response in a comparative study between nontreated and antifibrotic-treated IPF.

We postulate that in the near future, the use of blood biomarkers will be implemented in standard ILD care including IPF for diagnostic, prognostic and early predictive purposes. Possibly, decision making on treatments regiments in ILD will be based on clinical parameters including the outcomes of these predictive blood biomarkers.

\section{Conclusion}

In conclusion, we demonstrated that serum CA 15-3 could be useful as a minimally invasive, follow-up biomarker for treatment-response in IPF. Furthermore, CA 15-3 predicts PFT outcome and survival.

\section{Financial \& competing interests disclosure}

The authors have no relevant affiliations or financial involvement with any organization or entity with a financial interest in or financial conflict with the subject matter or materials discussed in the manuscript. This includes employment, consultancies, honoraria, stock ownership or options, expert testimony, grants or patents received or pending, or royalties.

No writing assistance was utilized in the production of this manuscript. 
Summary points

- Idiopathic pulmonary fibrosis (IPF) is a progressive interstitial lung disease (ILD) of unknown cause with a heterogeneous prognosis.

- Blood biomarkers such as serum pneumoproteins have a predictive value in IPF as they reflect the process of remodeling and injury of the lungs.

- The epitope cancer antigen 15-3 (CA 15-3) is located at the mucin 1 protein, expressed at alveolar type II pneumocytes. Serum CA 15-3 has been thoroughly investigated as a prognostic blood biomarker in various fibrotic ILDs including rheumatoid arthritis-ILD, systemic sclerosis-ILD and hypersensitivity pneumonitis.

- CA 15-3 is used a prognostic baseline biomarker in IPF, but its value during follow-up is unknown.

- The current study evaluated the prognostic value of CA 15-3 during 1-year follow-up in IPF patients using antifibrotic therapy at the St Antonius Hospital in Nieuwegein, The Netherlands.

- A repeated measures linear mixed model was used to analyse the associations between CA 15-3 and pulmonary function test (PFT) values over time. Survival was evaluated using Kaplan-Meier curves.

- A total of 132 IPF patients, predominately males (75.8\%) with mean age 68.6 years (70 pirfenidone-treated, 62 nintedanib-treated) were included in the study. Mean follow-up duration was 47.0 months.

- Baseline CA 15-3 values were elevated in all IPF patients (median: $56 \mathrm{kU} / \mathrm{l}$ ).

- Increased baseline and follow-up CA 15-3 measurements were inversely associated with corresponding forced vital capacity (estimate: $-5.21 ; p<0.001$ ) and diffusing capacity of the lung for carbon monoxide (estimate: -4.69 ; $\mathrm{p}<0.001)$ values.

- IPF patients with baseline and 6-month CA 15-3 levels above 58.5 (hazard ratio: $1.67 ; \mathrm{p}=0.031$ ) and $50.5 \mathrm{kU} / \mathrm{I}$ (hazard ratio: 2.99; $\mathrm{p}<0.001$ ), respectively, were associated with an impaired survival compared with IPF patients with lower levels.

- No significant differences were observed between pirfenidone- and nintedanib-treated IPF patients in associations between CA 15-3 and PFT and in survival rates.

- In conclusion, serum CA 15-3 is associated with PFT course during 1-year follow-up in IPF on antifibrotic treatment. Furthermore, higher baseline and follow-up CA 15-3 levels are related with poor survival.

- Serum CA 15-3 is a promising follow-up biomarker in IPF and could be implemented as a prognostic biomarker in IPF care.

\section{References}

Papers of special note have been highlighted as: $\bullet$ of interest; $\bullet \bullet$ of considerable interest

1. Travis WD, Costabel U, Hansell DM et al. An official American Thoracic Society/European Respiratory Society statement: update of the international multidisciplinary classification of the idiopathic interstitial pneumonias. Am. J. Respir. Crit. Care Med. 188(6), 733-748 (2013). http://www.ncbi.nlm.nih.gov/pubmed/24032382

2. Baumgartner KB, Samet JM, Stidley CA, Colby TV, Waldron JA. Cigarette smoking: a risk factor for idiopathic pulmonary fibrosis. Am. J. Respir. Crit. Care Med. 155(1), 242-248 (1997). http://www.ncbi.nlm.nih.gov/pubmed/9001319

3. Baumgartner KB, Samet JM, Coultas DB et al. Occupational and environmental risk factors for idiopathic pulmonary fibrosis: a multicenter case-control study. Am. J. Epidemiol. 152(4), 307-315 (2000). http://www.ncbi.nlm.nih.gov/pubmed/10968375

4. Jenkins RG, Simpson JK, Saini G et al. Longitudinal change in collagen degradation biomarkers in idiopathic pulmonary fibrosis: an analysis from the prospective, multicentre PROFILE study. Lancet Respir. Med. 3(6), 462-472 (2015).

https://linkinghub.elsevier.com/retrieve/pii/S221326001500048X

5. Hinz B, Phan SH, Thannickal VJ, Galli A, Bochaton-Piallat M-L, Gabbiani G. The myofibroblast. Am. J. Pathol. 170(6), 1807-1816 (2007). http://www.ncbi.nlm.nih.gov/pubmed/17525249

6. Palmucci S, Roccasalva F, Puglisi S et al. Clinical and radiological features of idiopathic interstitial pneumonias (IIPs): a pictorial review. Insights Imaging. 5(3), 347-364 (2014). http://link.springer.com/10.1007/s13244-014-0335-3

7. Nicholson AG, Colby TV, du Bois RM, Hansell DM, Wells AU. The prognostic significance of the histologic pattern of interstitial pneumonia in patients presenting with the clinical entity of cryptogenic fibrosing alveolitis. Am. J. Respir. Crit. Care Med. 162(6), 2213-2217 (2000). http://www.atsjournals.org/doi/abs/10.1164/ajrccm.162.6.2003049

8. Li X, Peng S, Wei L, Li Z. Relevance analysis of clinical and lung function parameters changing and prognosis of idiopathic pulmonary fibrosis. Int. J. Clin. Exp. Med. 7(12), 4759-4769 (2014). http://www.ncbi.nlm.nih.gov/pubmed/25663972

9. du Bois RM, Weycker D, Albera C et al. Six-minute-walk test in idiopathic pulmonary fibrosis: test validation and minimal clinically important difference. Am. J. Respir. Crit. Care Med. 183(9), 1231-1237 (2011). http://www.atsjournals.org/doi/abs/10.1164/rccm.201007-1179OC

10. Ley B, Ryerson CJ, Vittinghoff $\mathrm{E}$ et al. A multidimensional index and staging system for idiopathic pulmonary fibrosis. Ann. Intern. Med. 156(10), 684 (2012). http://www.ncbi.nlm.nih.gov/pubmed/22586007 
11. Raghu G, Collard HR, Egan JJ et al. An official ATS/ERS/JRS/ALAT statement: idiopathic pulmonary fibrosis: evidence-based guidelines for diagnosis and management. Am. J. Respir. Crit. Care Med. 183(6), 788-824 (2011). http://www.atsjournals.org/doi/abs/10.1164/rccm.2009-040GL

12. Karimi-Shah BA, Chowdhury BA. Forced vital capacity in idiopathic pulmonary fibrosis - FDA review of pirfenidone and nintedanib. N. Engl. J. Med. 372(13), 1189-1191 (2015). http://www.ncbi.nlm.nih.gov/pubmed/25806913

13. Miller MR. Does the use of per cent of predicted have any evidence base? Eur. Respir. J. 45(2), 322-323 (2015). http://erj.ersjournals.com/lookup/doi/10.1183/09031936.00199414

14. Kruit A, Gerritsen WBM, Pot N, Grutters JC, van den Bosch JMM, Ruven HJT. CA 15-3 as an alternative marker for KL-6 in fibrotic lung diseases. Sarcoidosis, Vasc. Diffus. Lung Dis. Off. J. WASOG. 27(2), 138-146 (2010).

http://www.ncbi.nlm.nih.gov/pubmed/21319596

-• Serum cancer antigen 15-3 (CA 15-3) was evaluated as a viable alternative to KL-6 for interstitial lung diseases (ILDs) with and without fibrosis, including idiopathic pulmonary fibrosis (IPF), sarcoidosis and hypersensitivity pneumonitis. Analysis showed a strong correlation between KL-6 and CA 15-3. In addition, both biomarkers performed best in identifying IPF and to differentiate between fibrotic and nonfibrotic ILD. This is the first study to describe CA 15-3 as an alternative biomarker for KL-6 and a possible marker for pulmonary fibrosis.

15. Prasse A, Probst C, Bargagli E et al. Serum CC-chemokine ligand 18 concentration predicts outcome in idiopathic pulmonary fibrosis. Am. J. Respir. Crit. Care Med. 179(8), 717-723 (2009). http://www.atsjournals.org/doi/abs/10.1164/rccm.200808-1201OC

16. Organ LA, Duggan AMR, Oballa E et al. Biomarkers of collagen synthesis predict progression in the PROFILE idiopathic pulmonary fibrosis cohort. Respir. Res. 20(1), 1-10 (2019).

17. Ishikawa N, Hattori N, Yokoyama A, Kohno N. Utility of KL-6/MUC1 in the clinical management of interstitial lung diseases. Respir. Investig. 50(1), 3-13 (2012). https://linkinghub.elsevier.com/retrieve/pii/S2212534512000020

18. Ballester B, Milara J, Cortijo J. Mucins as a new frontier in pulmonary fibrosis. J. Clin. Med. 8(9), 1447 (2019).

- An overview of the biological functions of mucins are described in this paper. Mucins are recognised as effectors in cell growth and tissue remodeling. These functions are compatible with the processes as seen in IPF. Therefore, mucins and epitopes of these mucins, including pneumoproteins, play a key role in the search for biomarkers for pulmonary fibrosis.

19. Matsuzawa $Y$, Kawashima T, Kuwabara R et al. Change in serum marker of oxidative stress in the progression of idiopathic pulmonary fibrosis. Pulm. Pharmacol. Ther. 32, 1-6 (2015). http://www.ncbi.nlm.nih.gov/pubmed/25862941

20. Ohshimo S, Ishikawa N, Horimasu Y et al. Baseline KL-6 predicts increased risk for acute exacerbation of idiopathic pulmonary fibrosis. Respir. Med. 108(7), 1031-1039 (2014). https://linkinghub.elsevier.com/retrieve/pii/S0954611114001541

21. Bergantini L, Bargagli E, Cameli P et al. Serial KL-6 analysis in patients with idiopathic pulmonary fibrosis treated with nintedanib. Respir. Investig. 57(3), 290-291 (2019). http://www.ncbi.nlm.nih.gov/pubmed/30799155

22. Okada M, Suzuki K, Nakanishi T, Nakashima M. Serum levels of KL-6 are positively correlated with those of CA 15-3 in patients with interstitial pneumonia associated with collagen diseases [1]. Respirology 11, 509-510 (2006).

23. Ri G, Ohno S, Yamamoto T et al.Serum levels of CA 15-3, KL-6 and BCA225 are positively correlated with each other in the general population. https://www.ncbi.nlm.nih.gov/pubmed/19846980

24. Molina R, Barak V, Van Dalen A et al. Tumor markers in breast cancer - European group on tumor markers recommendations. Tumor Biol. 26, 281-293 (2005).

25. Fu Y, Li H. Assessing clinical significance of serum CA 15-3 and carcinoembryonic antigen (CEA) levels in breast cancer patients: a meta-analysis. Med. Sci. Monit. 22, 3154-3162 (2016).

26. Ricci A, Mariotta S, Bronzetti E et al. Serum CA 15-3 is increased in pulmonary fibrosis. Sarcoidosis, Vasc. Diffus. Lung. Dis. Off. J. WASOG. 26(1), 54-63 (2009). http://www.ncbi.nlm.nih.gov/pubmed/19960789

-. CA 15-3 levels were evaluated in IPF patients and compared with sarcoidosis, systemic sclerosis (Ssc) and healthy subjects. CA 15-3 levels were significantly higher in IPF subjects compared with the other groups. Furthermore, increased levels showed associations with decreased pulmonary function outcome and fibrotic findings on high-resolution computed tomography.

27. Wang T, Zheng XJ, Ji YL, Liang ZA, Liang BM. Tumour markers in rheumatoid arthritis-associated interstitial lung disease. Clin. Exp. Rheumatol. 34(4), 587-591 (2016).

28. Baldus SE, Engelmann K, Hanisch FG. MUC1 and the MUCs: a family of human mucins with impact in cancer biology. Crit. Rev. Clin. Lab. Sci. 41, 189-231 (2004). http://www.tandfonline.com/action/journalInformation?journalCode=ilab20

29. Bennett D, Salvini M, Fui A et al. Calgranulin B and KL-6 in bronchoalveolar lavage of patients with IPF and NSIP. Inflammation 42(2), 463-470 (2019). http://www.ncbi.nlm.nih.gov/pubmed/30680696

30. Celeste S, Santaniello A, Caronni M et al. Carbohydrate antigen 15.3 as a serum biomarker of interstitial lung disease in systemic sclerosis patients. Eur. J. Intern. Med. 24(7), 671-676 (2013). http://www.ncbi.nlm.nih.gov/pubmed/23697634

31. De Luca G, Bosello SL, Berardi G et al. Tumour-associated antigens in systemic sclerosis patients with interstitial lung disease: association with lung involvement and cancer risk. Rheumatol (United Kingdom). 54(11), 1991-1999 (2015). 
32. Marzano AV, Morabito A, Berti E, Caputo R. Elevated circulating ca 15.3 Levels in a subset of systemic severe lung involvement [13]. Arch. Dermatol. 134, 645 (1998).

- In this study, associations between CA 15-3 levels, radiological findings and pulmonary function outcome were assessed in Ssc-ILD patients. Subjects with increased CA 15-3 levels showed lower forced vital capacity values, higher interstitial score and alveolar score compared with Ssc-ILD patients without elevated CA 15-3 levels.

33. Moll SA, Wiertz IA, Vorselaars ADM, Ruven HJT, van Moorsel CHM, Grutters JC. Change in serum biomarker CA 15-3 as an early predictor of response to treatment and survival in hypersensitivity pneumonitis. Lung 198(2), 385-393 (2020).

http://link.springer.com/10.1007/s00408-020-00330-9

-. Associations between CA 15-3 values and pulmonary function tests were evaluated in patients with fibrotic and nonfibrotic hypersensitivity pneumonitis. It was demonstrated that early CA 15-3 change correlated inversely with future pulmonary function change. This is the first study to describe the value of CA 15-3 as a predictive biomarker in fibrotic lung disease.

34. Szekanecz É, Szucs G, Szekanecz Z et al. Tumor-associated antigens in systemic sclerosis and systemic lupus erythematosus: associations with organ manifestations, immunolaboratory markers and disease activity indices. J. Autoimmun. 31(4), 372-376 (2008).

35. Sargin G, Köse R, Şentürk T. Tumor-associated antigens in rheumatoid arthritis interstitial lung disease or malignancy? Arch. Rheumatol. 33(4), 431-437 (2018).

36. Rusanov V, Kramer MR, Raviv Y, Medalion B, Guber A, Shitrit D. The significance of elevated tumor markers among patients with idiopathic pulmonary fibrosis before and after lung transplantation. Chest 141(4), 1047-1054 (2012). http://www.ncbi.nlm.nih.gov/pubmed/21940773

37. King TE, Bradford WZ, Castro-Bernardini $S$ et al. A Phase III trial of pirfenidone in patients with idiopathic pulmonary fibrosis. $N$. Engl. J. Med. 370(22), 2083-2092 (2014). http://www.ncbi.nlm.nih.gov/pubmed/24836312

38. Raghu G, Remy-Jardin M, Myers JL et al. Diagnosis of idiopathic pulmonary fibrosis. An official ATS/ERS/JRS/ALAT clinical practice guideline. Am. J. Respir. Crit. Care Med. 198(5), e44-e68 (2018). http://www.ncbi.nlm.nih.gov/pubmed/30168753

- Clinical recommendations for the diagnostic criteria of IPF are described in this recent update of the ATS/ERS 2018 IPF guidelines. Previously defined patterns of usual interstitial pneumonia (UIP; UIP, possible UIP and inconsistent with UIP) have been revised to four categories of UIP (UIP, probable UIP, indeterminate UIP and alternative diagnosis) in order to enhance diagnostic work-up of IPF.

39. Bergantini L, Bianchi F, Cameli P et al. Prognostic biomarkers of sarcoidosis: a comparative study of serum chitotriosidase, ACE, Lysozyme, and KL-6. Dis. Markers 2019, 1-7 (2019). http://www.ncbi.nlm.nih.gov/pubmed/30944672

40. Wakamatsu K, Nagata N, Kumazoe H et al. Prognostic value of serial serum KL-6 measurements in patients with idiopathic pulmonary fibrosis. Respir. Investig. 55(1), 16-23 (2017). http://www.ncbi.nlm.nih.gov/pubmed/28012488

- The prognostic value of serial KL-6 measurements was evaluated in untreated IPF. Patient with increased serum KL-6 levels during follow-up demonstrated deeper declines of pulmonary function test (forced vital capacity) and worse survival outcome compared with patients without KL-6 increase. This study emphasizes the predictive value of a mucin-1 epitope in IPF.

41. Ishii H, Kushima H, Kinoshita Y, Fujita M, Watanabe K. The serum KL-6 levels in untreated idiopathic pulmonary fibrosis can naturally decline in association with disease progression. Clin. Respir. J. 12(9), 2411-2418 (2018). http://www.ncbi.nlm.nih.gov/pubmed/30015394

42. Wémeau-Stervinou L, Perez T, Murphy C, Polge A-S, Wallaert B. Lung capillary blood volume and membrane diffusion in idiopathic interstitial pneumonia. Respir. Med. 106(4), 564-570 (2012). http://www.ncbi.nlm.nih.gov/pubmed/22221584

43. Sakai M, Kubota T, Ohnishi H, Yokoyama A. A novel lung injury animal model using KL-6-measurable human MUC1-expressing mice. Biochem. Biophys. Res. Commun. 432(3), 460-465 (2013). https://linkinghub.elsevier.com/retrieve/pii/S0006291X13002428

- In bleomycine-induced and lipopolysaccharide-induced murine lung models, serum KL-6 levels in bronchoalveolar lavage fluid were examined and investigated whether KL-6 reflects the degree of lung injury. An increase in serum KL-6 levels was observed in the inflammatory phase of the bleomycine-induced murine model, which was thought to be associated with the disruption of alveolar-capillary barrier. Thus, KL-6 was demonstrated to be a representative marker for increased alveolar-capillary permeability.

44. Nakayama M, Bando M, Araki K et al. Physical activity in patients with idiopathic pulmonary fibrosis. Respirology 20(4), 640-646 (2015).

45. Sakamoto K, Taniguchi H, Kondoh Y et al. Serum KL-6 in fibrotic NSIP: correlations with physiologic and radiologic parameters. Respir. Med. 104(1), 127-133 (2010).

46. Maher TM, Oballa E, Simpson JK et al. An epithelial biomarker signature for idiopathic pulmonary fibrosis: an analysis from the multicentre PROFILE cohort study. Lancet Respir. Med. 5(12), 946-955 (2017).

47. Shiota Y, Furuya K, Kitade M, Ueda N. CEA and CA19-9 in BALF from patients with idiopathic interstitial pneumonia. https://www.ncbi.nlm.nih.gov/pubmed/2615104.

48. Wong RCW, Klingberg S, Wilson R. CA 15-3 and cancer associated serum antigen assays are alternatives to the KL-6 assay for measuring serum MUC-1 levels in patients with interstitial. https: 
//www.ncbi.nlm.nih.gov/pubmed/?term=CA15-3+and+cancer+associated+serum+antigen+assays+are+alternatives+to+the+KL-6+assay +for+measuring+serum+MUC-1+levels+in+patients+with+interstitial+lung+disease+associated+with+polymyositis\%2Fdermatomyositis

49. Lappi-Blanco E, Mäkinen JM, Lehtonen S et al. Mucin-1 correlates with survival, smoking status, and growth patterns in lung adenocarcinoma. Tumor Biol. 37(10), 13811-13820 (2016).

50. Juarez MM, Chan AL, Norris AG, Morrissey BM, Albertson TE. Acute exacerbation of idiopathic pulmonary fibrosis-a review of current and novel pharmacotherapies. J. Thorac. Dis. 7(3), 499-519 (2015). http://www.ncbi.nlm.nih.gov/pubmed/25922733

51. Distler O, Highland KB, Gahlemann $\mathrm{M}$ et al. Nintedanib for systemic sclerosis-associated interstitial lung disease. N. Engl. J. Med. 380(26), 2518-2528 (2019). http://www.nejm.org/doi/10.1056/NEJMoa1903076

52. Flaherty KR, Wells AU, Cottin V et al. Nintedanib in progressive fibrosing interstitial lung diseases. N. Engl. J. Med. 381(18), 1718-1727 (2019). http://www.nejm.org/doi/10.1056/NEJMoa1908681 
